\title{
Cooperativas rurales y sistemas de gestión de calidad como estrategia en cadenas productivas agrarias en Nicaragua
}

\author{
Isabel Ortiz-Marcos* \\ José Ramón Cobo Benita* \\ Carlos Mataix Aldeanueva* \\ Luz Fernández García* \\ Universidad Politécnica de Madrid. Madrid, España
}

Recibido: 5/3/2012 / Aprobado: 1/4/2012

\begin{abstract}
RESUMEN: Este artículo muestra cómo diferentes modelos cooperativos y sistemas de gestión de la calidad pueden cumplir diversas funciones en la integración y consolidación de cadenas productivas agrarias para mejorar las condiciones de vida en el mundo rural. El caso desarrollado por el Grupo de Cooperación en Organización, Calidad y Medio Ambiente (GOCMA) de la Universidad Politécnica de Madrid para una cooperativa de "segundo piso", es un ejemplo práctico de coordinación y colaboración en cooperativas de Nicaragua.
\end{abstract}

Palabras clave: Cooperativas rurales / sistemas de gestión de calidad / cadenas productivas agrarias

\section{Rural cooperatives and quality management in agricultural production chains in Nicaragua}

ABstract: This paper shows how different cooperative models and quality management systems can play different roles in the integration and consolidation of farming production chains to improve the standard of living in rural parts of the world. The case developed by Madrid Polytechnic University's group for co-operation in organisation, quality and environment for a cooperative of "second level" is an authentic practical example of coordination and collaboration between cooperatives in Nicaragua.

Keywords: Rural cooperatives / quality management systems / farming production chains

* Correos electrónicos: Isabel.ortiz@upm.es, joseramon.cobo@upm.es, carlos.mataix@upm.es, lfernandezg@upm.es. 


\section{CARACTERÍSTICAS PRODUCTIVAS DEL MEDIO RURAL EN AMÉRICA CENTRAL}

Una gran parte de los campesinos de la región centroamericana viven por debajo del umbral de la pobreza. En su mayoría son pequeños productores y minifundistas. En Nicaragua, por ejemplo, estos constituyen las tres cuartas partes del total de los hogares del ámbito rural (PNUD, 2010).

Los rendimientos medios por unidad de superficie del sector agropecuario en la región no han mejorado mucho en los últimos 30 años. Solamente se incrementa en las llanuras y regiones más fértiles, debido a la utilización de mayores cantidades de abonos y productos fitosanitarios (Banco Mundial, 2010). La falta de acceso a semillas certificadas, asistencia técnica y a recursos financieros para introducir mejoras en las fincas son, entre otras, causas endémicas de esta situación.

En todos los países predomina la agricultura temporal y el uso de tecnologías manuales. En Nicaragua, el país con menos tractores y animales de tiro por unidad de superficie agropecuaria de toda Centroamérica, más del $75 \%$ de la producción de granos básicos se cultiva manualmente; según las cifras del Banco Central de Nicaragua (2010), los niveles de uso de insumos y bienes de capital para la agricultura han representado, en los últimos 6 años, menos del 5\% del PIB agropecuario. La utilización de sistemas de riego se limita a los grandes finqueros. La mayoría de los pequeños productores dependen de la estacionalidad de las lluvias para cultivar.

El sector agroindustrial tiene un escaso desarrollo y está poco articulado con el resto de los sectores económicos. En Nicaragua, el 92\% de la agroindustria es básica, esto es, dedicada a producciones que requieren un procesamiento relativamente sencillo de productos primarios, tales como café, azúcar o leche.

Si bien una agroindustria de productos más elaborados tiene un gran potencial de creación de valor agregado, las unidades involucradas en este sector son pocas y con bajos niveles de capitalización, debido, principalmente, a la inexistencia de políticas sectoriales que la promuevan y a la falta de capital e iniciativa empresarial.

Las cadenas de comercialización de la región suelen estar en manos de un amplio sector de intermediarios. Además, los costes de transpor- 
te son muy elevados, por lo que los productores a menudo son incapaces de trasladar sus productos a mercados locales o regionales.

Debido a que para las familias campesinas el acceso al crédito es difícil, cuando no imposible, estas con frecuencia se ven obligadas, como estrategia para obtener capital a corto plazo, a vender a futuro su cosecha, por lo general, a la mitad del precio de mercado.

A todo lo anterior se une la limitada capacidad del Estado para destinar recursos al medio rural, lo cual ha repercutido en la disminución progresiva de los servicios sociales, como educación, salud o agua potable, y de producción: financieros, asistencia técnica, infraestructuras básicas, etcétera (Londoño \& Mataix, 2001).

A menudo existe un vacío legal en cuanto a la seguridad de la tenencia y el acceso a la tierra, ya que no existe reconocimiento de los derechos que sobre ella tienen las comunidades indígenas. Las formas de acceso temporal a la tierra como alquiler o contratos son poco eficaces y seguras, y existen problemas de titularidad.

En un contexto regional determinado, la actividad del sector de las pequeñas unidades de producción agraria puede analizarse como parte de un sistema más amplio de producción y distribución. Generalmente, salvo en el caso de grandes corporaciones integradas verticalmente, en dichos sistemas participan diferentes actores, entre quienes se establecen transacciones basadas en relaciones de cooperación y de competencia. De hecho, todos los campesinos cuya actividad va más allá de la producción para el autoconsumo están integrados, de un modo u otro, en cadenas productivas agrarias más amplias.

Una cadena productiva agraria integra, al menos, tres etapas: producción, transformación y distribución. El análisis de la agregación de valor a lo largo de la cadena, de la retribución de cada actor, de sus relaciones de poder, y del grado de coordinación entre las diferentes etapas, explica el nivel de éxito relativo de la cadena.

Pero esto no es exclusivo del ámbito agroindustrial. En una economía globalizada, prácticamente en todos los sectores, agrarios o no, el diseño y la gestión de las cadenas logísticas o cadenas de suministro constituye un aspecto decisivo en la competitividad y sostenibilidad de las organizaciones productivas. No se trata solo de que cada parte desempeñe bien su función, también es necesario que exista un diseño de 
la actividad de las partes que tenga en cuenta el sistema en su conjunto (Carrasco, 2000).

En el caso que nos ocupa, el de las cadenas agrarias en Centroamérica, puede observarse cómo los problemas descritos anteriormente afectan a todas y cada una de las etapas que conforman las cadenas. Como resultado, las cadenas productivas agrarias de la región tienen graves problemas para competir en mercados abiertos y presentan una marcada asimetría en las relaciones entre sus integrantes, constituyendo el de los campesinos productores primarios su eslabón más débil y, por lo tanto, el peor retribuido.

\section{LA PROMOCIÓN DEL COOPERATIVISMO COMO ESTRATEGIA PARA LA INTEGRACIÓN DE CADENAS PRODUCTIVAS AGRARIAS}

La integración de los pequeños productores en sistemas cooperativos capaces de crear y aprovechar economías de escala, añadiendo valor a las producciones individuales y facilitando el acceso a determinados servicios, constituye una estrategia que, con mayor o menor éxito, ha formado parte de numerosos programas de cooperación para el desarrollo.

A continuación se expone cómo, desde la perspectiva de la integración paulatina de los pequeños productores en las cadenas productivas agrarias, diferentes modelos cooperativos pueden cumplir diferentes funciones.

\subsection{Economía de subsistencia}

En este escenario, el productor no tiene excedentes y destina toda la producción al consumo familiar. No está dentro del mercado, no pretende obtener ingresos de su actividad productiva y trabaja como asalariado; es muy conservador y no arriesga.

Es el escenario propio de una gran parte de la población rural pobre de Centroamérica.

\subsection{Productor aislado}

Realiza una actividad económica para obtener ingresos. No tiene capacidad de diversificación ni de rentabilizar toda la tierra (figura 1). 
En ocasiones accede a sistemas informales de crédito, normalmente facilitados por el proveedor de insumos.

Tiene un débil nexo con el mercado y vende su producción a intermediarios que habitualmente son los mismos que le proporcionan los insumos. Encuentra difícil acceso a nuevas tecnologías y usa tecnología manual.

Figura 1

Productor aislado

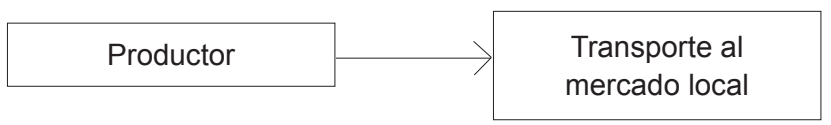

Elaboración propia.

\subsection{Cooperativa}

Está constituida por productores que generalmente tienen tierra en propiedad y que trabajan individualmente, asociándose a otros en una cooperativa (figura 2). El crédito y la tecnología están entre sus prioridades. Utilizan maquinaria agrícola y, en algunos casos, tecnología de riego.

El acceso a insumos y tecnología les da la oportunidad de obtener un incremento en los rendimientos. Normalmente la cooperativa se centra en la venta de un único producto y recibe algún tipo de asistencia técnica.

Figura 2

Cooperativa

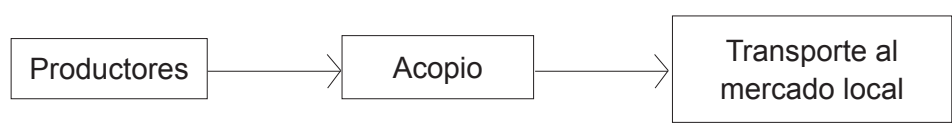

Elaboración propia.

Por lo general, estas cooperativas tienen infraestructura para almacenar la producción de sus socios, y en el caso de cooperativas fuertes, con muchos socios, negocian precios en el mercado local, nacional e incluso internacional. 
En el paso a esta etapa, si la cooperativa ha tenido éxito habrá generado y aprovechado economías de escala, obteniendo mejores precios por la venta de los productos y reduciendo los costes de compra de insumos. Pero para constituir la cooperativa habrá sido necesario que se den cita determinados factores culturales y de disponibilidad de medios económicos y humanos.

\subsection{Cooperativa + valor añadido}

En este contexto, el productor alcanza un mayor nivel de integración y presencia en la cadena productiva (figura 3). La transformación puede, en muchas ocasiones, consistir simplemente en una selección, limpieza, envasado y etiquetado de sus productos. En otras, se trata del procesamiento de la materia prima; por ejemplo, una fábrica de vinagre de frutas o una fábrica de concentrado para animales. Pero en todos los casos, "transformación" significa "adición de valor", lo cual redunda en una mejora de los ingresos de los productores, a la vez que significa una mayor capacidad de afrontar las oscilaciones de los mercados.

\section{Figura 3}

\section{Cooperativa + valor añadido}

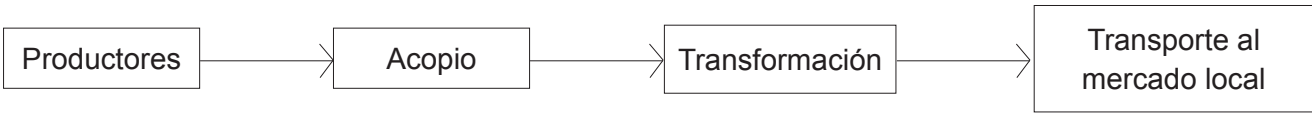

Elaboración propia.

Sin embargo, para llegar a esta fase se requieren condiciones tales como financiación adecuada y personal cualificado, que en muchas regiones difícilmente se reúnen sin apoyo de instituciones promotoras.

\subsection{Integración de cadenas productivas agrarias + valor añadido + línea de productos: Cadena de comercialización}

En este escenario, las cooperativas de ámbito local deciden integrarse en cooperativas de mayor rango (llamadas cooperativas de "segundo grado" o "segundo piso") capaces, por un lado, de prestar servicios especializados que requieren mayor escala, tales como procesamiento de productos, envasado o asistencia técnica especializada, y por otro lado, 
de coordinar y planificar la producción de las diferentes cooperativas en función de las necesidades de los mercados (figura 4).

La integración de cooperativas proporciona la posibilidad de crear cadenas multiproducto, que permiten diseñar y poner en práctica estrategias de mercado propias, mediante la creación de marcas que penetran directamente en los mercados de consumidores.

Figura 4

Integración de cadenas productivas agrarias + valor añadido + línea de productos: cadena de comercialización

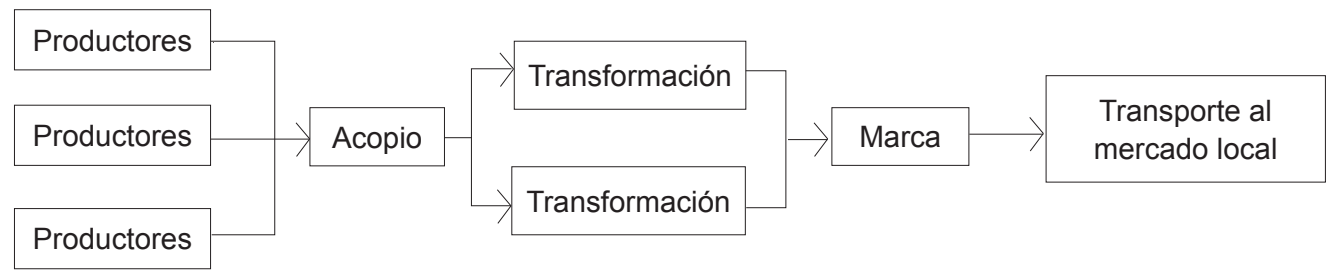

Elaboración propia.

En definitiva, en este escenario los productores participan, directa $o$ indirectamente, en todas las fases de la cadena productiva. Se trata de un paso decisivo hacia la modernización del medio productivo rural.

Pero se trata también del escenario más difícil de alcanzar, que requiere actuar simultánea y coordinadamente en diversos frentes. En este sentido, la cooperativa objeto de estudio — resultado de una experiencia de promoción de cooperativas rurales en la que están comprometidas organizaciones no gubernamentales de desarrollo (ONGD) nicaragüenses y sus contrapartes de otros países - sirve para ilustrar cómo se pueden coordinar cooperativas para la creación y la gestión de cadenas productivas agrarias complejas. El grupo de investigación para la cooperación al desarrollo en organización, calidad y medioambiente de la Universidad Politécnica de Madrid acompañó a estas organizaciones en el desarrollo de los sistemas de gestión necesarios para hacer frente al crecimiento de las cooperativas asociadas a este movimiento. 


\section{CASO DE ESTUDIO: COOPERATIVA DE "SEGUNDO PISO" EN NICARAGUA}

La cooperativa objeto de estudio reúne las siguientes características:

- Es "de segundo piso": presta servicios a un conjunto de cooperativas (o uniones de cooperativas) de productores rurales.

- Es auxiliar: el grado de integración de las cooperativas asociadas es parcial (no dependen exclusivamente de ella).

- Es de implantación nacional: sus cooperativas asociadas se encuentran en diferentes regiones de Nicaragua.

- Es multiactiva o multifuncional: presta diversos tipos de servicios a las cooperativas asociadas.

Los servicios que ofrece a sus socios son:

- Apoyo en la producción y acopio: financiación para crear un fondo de crédito para acopio y procesamiento. El capital de acopio y transformación permitirá a la cooperativa de "segundo piso" comprar la producción a las cooperativas y aumentar el volumen de producto para tener una mayor capacidad de oferta y poder firmar contratos comerciales con lo que se asegura el abastecimiento continuado de una cierta cantidad de productos.

- Asistencia técnica para la agregación de valor en:

- Certificación de productos orgánicos o ecológicos, registro de productos.

- El proceso productivo, en colaboración con las universidades nicaragüenses y de otros países.

- El envasado y etiquetado de los productos.

- Las áreas gerencial, productiva y administrativa de las organizaciones.

- Apoyo en la comercialización de productos:

- Acompañamiento en las operaciones de transacción, manejo de las bases de datos de precios y mercados, información y capacitación para facilitar el acceso conjunto a mercados locales y de exportación.

- Promoción y comercialización de la producción a través de una marca registrada, cuyos productos incluyen atributos de valor social, ambiental, de calidad y de salud. 
Las organizaciones campesinas asociadas a esta cooperativa forman una asamblea compuesta por socios delegados de las organizaciones de base, que toman decisiones en forma democrática. Los excedentes económicos que se consiguen se devuelven a los productores en forma de primas poscomercialización. Con un porcentaje del precio de venta de los productos agrícolas transformados o semitransformados se pretende cubrir la capacidad operativa de la empresa central, de forma que pueda reducir paulatinamente el apoyo de organismos externos.

Las organizaciones socias producen cultivos anuales (ajonjolí, fríjol, maíz, arroz, plantas medicinales), perennes (café, marañón o anacardo) y productos pecuarios (miel, lácteos). Los granos básicos se destinan a mercados locales y los cultivos de exportación se producen con técnicas agroecológicas y se certifican como productos orgánicos por la certificadora BioLatina, autorizada por la agencia alemana DAR, lo que permite su comercialización en mercados europeos.

Entre las características que han hecho de esta cooperativa un modelo de integración de cadenas productivas agrarias atractivo para los asociados (seis organizaciones de primer y segundo piso) y las organizaciones aliadas (hasta la fecha más de cincuenta organizaciones campesinas y empresas propiedad de trabajadores, que articulan un universo de más de 15.000 familias) se pueden señalar la siguientes:

- Independencia de la estructura central.- Todas las organizaciones socias tienen sus propias capacidades gerenciales, o se establece como objetivo la dotación paulatina de estas capacidades y su fortalecimiento administrativo y operativo. Además, todas ellas tienen capacidad legal (licencias, registros y permisos) para establecer relaciones autónomas con los mercados, incluso los de exportación; es decir, la empresa central basa su existencia en la generación de eficiencia (consecución de mejores precios y mercados) y no en el monopolio de capacidades o de información.

- Articulación vertical flexible y transparente.- La cooperativa de "segundo piso" objeto de estudio no obliga a las organizaciones asociadas a vender su producción a través de la empresa central. La negociación con los compradores de la producción agrícola integrada se hace con la participación de las organizaciones locales, con lo cual estas acceden a la información sobre precios y mercados. La empresa cooperativa suscribe contratos detallados con las organizaciones socias o aliadas, donde especifican los compromisos de las partes entre sí y con terceros. 
- Limitación del poder de la dirección y el aparato técnico.- El aparato técnico de la empresa es pequeño, en relación con el tamaño de la suma de sus asociados. Los activos (tierras, bodegas, equipos y capital de producción) están mayoritariamente en manos de las organizaciones locales socias, lo que obliga al consenso a la hora de su uso colectivo; por ejemplo, a la hora de utilizar garantías para la adquisición de créditos.

- La transferencia de riesgos y beneficios.- Los riesgos derivados de la producción agrícola recaen en las organizaciones locales. Los riesgos derivados de la comercialización recaen en la estructura central. Generalmente, esta cooperativa establece precios de garantía de compra superiores a los costos de producción y a los del mercado local. Las operaciones de acopio se realizan pagando al productor esos precios de garantía en el momento de la entrega del producto. Si existen excedentes en la comercialización, estos se devuelven al productor en forma de prima poscomercialización, descontando los costos del proceso y los porcentajes fijos para el mantenimiento de los aparatos administrativos y técnicos de las organizaciones (entre el 2 y el $5 \%$ del precio de venta final para la empresa central y la organización local).

\section{INTRODUCCIÓN DE SISTEMAS DE GESTIÓN DE LA CALIDAD EN LAS COOPERATIVAS CAMPESINAS}

Analizadas las características del movimiento cooperativista para la integración de pequeños productores en cadenas productivas agrarias, la empresa central de servicios identificó la necesidad de preparar y mejorar los sistemas de gestión para hacer frente al crecimiento de las cooperativas asociadas. En este sentido, se detectaron varias carencias o dificultades:

- Para poder exportar los productos a otros mercados se les exige demostrar su calidad a través de sellos de certificación de su sistema de producción y de sus propios productos.

- Las entidades promotoras requieren una mejora de sus propios sistemas de gestión con el objeto de realizar una labor de apoyo cada día más eficaz y eficiente.

En este contexto, la cooperativa de "segundo piso" objeto de estudio puso en funcionamiento una fábrica de descortezado de ajonjolí en Po- 
soltega. En ella se procesa el ajonjolí de las cooperativas productoras que componen esta cooperativa, obteniendo tres líneas de producto: ajonjolí descortezado, ajonjolí descortezado orgánico y aceite de ajonjolí. Los resultados desde el punto de vista industrial y comercial han sido muy prometedores, y han supuesto un gran impulso para la empresa central.

El sistema de producción tradicional utiliza la mano de obra campesina como principal insumo, durante la siembra, el cultivo, el emparve y el aporreo, obteniendo ajonjolí "natural sucio de campo". El 90\% de los socios de esta cooperativa y sus aliados está compuesto por familias campesinas que producen de 1 a 4 hectáreas de ajonjolí, bajo sistemas tradicionales y de bajos insumos. Un $10 \%$ de la producción es certificada como orgánica.

Mediante la introducción de sistemas de gestión de la calidad ISO 9001 se persigue mejorar la productividad (y en consecuencia los ingresos) de las 1.700 familias productoras pertenecientes a las cooperativas citadas mediante el fortalecimiento de los sistemas de organización, gestión e información de la cooperativa de "segundo piso". El primer paso para conseguir estos objetivos fue la preparación de la planta de descortezado de ajonjolí de Posoltega para hacer frente a una certificación de su sistema de gestión.

La consecución de este proyecto ha contribuido a reforzar su presencia en el medio rural al asegurar, a través de las acciones propuestas, un incremento de ingresos que les permite seguir viviendo en sus comunidades y mantener un equilibrio de población. Es la solución de los problemas, para la superación de las ineficiencias en la comercialización de productos agrícolas, que a su vez determinan el estado de pobreza de las familias campesinas. Con la introducción de estos sistemas de gestión, se consigue:

a) Facilitar la exportación a los productores de ajonjolí, marañón, fríjol, miel y café dadas las ventajas comparativas de estos cultivos frente a otros. Son productos rentables, se pueden sembrar bajo tecnologías tradicionales, los beneficiarios tienen una experiencia de décadas en su cultivo y existen mercados en expansión a nivel nacional e internacional (Europa, Japón y América del Norte).

b) Hacer posible que las organizaciones beneficiarias cuenten con volúmenes comercializables de estos productos, lo cual los hará más atractivos en los mercados. 
c) El fortalecimiento de las organizaciones participantes en el proyecto les permitirá proveer de mejores servicios a sus asociados, estar en comunicación permanente con los mercados y asumir convenios con instituciones de gobierno sectoriales (Instituto Nicaragüense de Tecnología Agropecuaria, Banco Central de Nicaragua), programas y cámaras de comercio.

La primera fase para la implantación del sistema de gestión consistió en la realización de un informe de diagnosis. Este informe se estructura de manera que se compara, para cada área de la planta de Posoltega, lo que hay con lo que debería haber para cumplir con los requisitos de la norma ISO 9001:2008; se proponen las mejoras a implementar así como la documentación necesaria. Con esta documentación se debe establecer y mantener un sistema de gestión de la calidad que permita y facilite la estandarización de procesos, sirva como medida de calidad, promueva la mejora continua y permita obtener la certificación del sistema de gestión de la calidad. Para ello se entrevistó a miembros de la organización de los ámbitos técnico, administrativo y gerencial.

La diagnosis realizada alcanzó los siguientes objetivos:

- Establecer el grado de adecuación de la organización, funciones, documentos y actividades con los requisitos de la normativa de gestión de calidad ISO 9001:2008.

- Identificar los procesos estratégicos, los procesos operativos (que proporcionan valor al cliente) y los procesos de apoyo (necesarios para el funcionamiento de los procesos operativos y para la gestión de la empresa) que son llevados a cabo en la planta de Posoltega.

- Elaborar el mapa de procesos de la planta de Posoltega.

- Obtener la información necesaria para completar el manual de calidad y los procedimientos generales que constituyen la documentación básica del sistema de gestión de calidad.

- Evaluar los requerimientos de los edificios de la planta (piso, suelo, techo, iluminación, ubicación, diseño, alrededores, puertas y ventanas, ventilación).

- Evaluar los requerimientos de instalaciones sanitarias (abastecimiento de agua, lavados de manos, drenajes, etcétera).

- Evaluar los requerimientos sobre manejo y disposición de los desechos líquidos y sólidos. 
- Evaluar los programas de limpieza y desinfección.

- Evaluar el control de plagas.

- Evaluar los equipos y utensilios.

- Evaluar las prácticas del personal.

- Evaluar el control de los procesos de la planta y la producción.

- Evaluar el almacenamiento y la distribución de los productos procesados.

Una vez recogida la información necesaria, se desarrollaron los procedimientos generales (elaboración y control de la documentación; realización de auditorías internas; tratamiento de las no conformidades; acciones correctoras y preventivas; control de los registros; tratamiento de quejas, sugerencias y reclamaciones; control de indicadores y acciones de mejora continua) y los procedimientos operativos necesarios (control de equipos; desarrollo de nuevos productos; gestión administrativa; gestión de los almacenes; gestión de compras y selección de proveedores; gestión de personal: contratación, formación, capacitación y despido; producción y control de puntos críticos y medida de satisfacción del cliente).

Tras el análisis realizado en la planta de descortezado de Posoltega se concluyó que se debían aplicar las siguientes disposiciones:

- Mejorar los aspectos medioambientales: análisis del agua de entrada, tratamiento del agua de salida, etcétera.

- Favorecer los aspectos relacionados con el ambiente de trabajo de la planta: limpieza del equipamiento de la planta, uso de uniforme, mascarilla, protector contra el ruido.

- Establecer un mantenimiento preventivo de los equipos, para evitar paradas durante el proceso de producción.

- Calibrar y verificar los equipos de medida.

- Guardar y mantener registros de todo el personal involucrado en el funcionamiento de la planta.

También se desarrolló el manual de calidad, donde se establece la política de calidad, la organización de la planta, las funciones y responsabilidades, así como el sistema de gestión diseñado.

Una vez identificadas las necesidades para un correcto funcionamiento del sistema de gestión, se tomó contacto con la Universidad 
Nacional de Ingeniería en Nicaragua (UNI), lo que permitió realizar un estudio para optimizar el consumo de energía y combustibles, como parte del proceso de mejora continua productiva y ambiental previsto.

Esta auditoría energética evalúa las oportunidades de optimización de los consumidores energéticos, considerando las prácticas de utilización, condiciones de operaciones, eficiencia y administración de la carga y de la energía. El objetivo final es desarrollar un programa de ahorro energético que contemple la implementación de cambios de proceso, prácticas o tecnologías que aumenten el rendimiento energético. Este programa incluye medidas que han sido evaluadas técnica, económica y ambientalmente; además, implica la capacitación del personal para la sostenibilidad del sistema de control y administración de la energía que se desarrolla para el monitoreo de resultados.

Otra aportación importante realizada por parte de la UNI ha sido el análisis del sistema de registro e inventarios para el control de la producción. Este componente tiene como fin crear un método de registro que permita a la empresa generar información para la toma de decisiones, a través del análisis del comportamiento de la producción, como de indicadores de control que se consideren relevantes. Permitirá también una mejor administración y la creación de fichas de registro de mantenimiento de los equipos, con el fin de crear la base para el manejo de la información indispensable para la optimización del proceso productivo.

Actualmente, la fábrica de Posoltega se está preparando en el desarrollo de la capacidad interna que le permita implementar la normativa más relevante para el cumplimiento de las exigencias del mercado de productos alimenticios. Esto es, la aplicación de buenas prácticas de manufacturas (obligatorio a nivel nacional para las empresas procesadoras de alimentos) y el sistema de análisis de riesgos y puntos críticos de control (HACCP, por sus siglas en inglés). Ambos requerimientos son los sistemas más reconocidos y comprobados a nivel internacional para asegurar la inocuidad y sanidad de los alimentos y cuentan con metodologías y prácticas de aplicación que las empresas desarrollan en forma sistemática en el diario funcionamiento de sus operaciones.

De este modo, y en colaboración con el Centro de Producción Más Limpia de Nicaragua, se está desarrollando la capacidad personal de los trabajadores de la planta de Posoltega para implementar el sistema de inocuidad alimenticia que garantice su desarrollo paulatino y el 
cumplimiento de las normas de seguridad, higiene e inocuidad de los alimentos, exigidas en los mercados nacional e internacional.

En este sentido, se plantea capacitar al personal de la empresa en el desarrollo del HACCP, identificar las fortalezas y debilidades de la empresa en el cumplimiento de los requerimientos básicos de inocuidad e higiene, definir acciones prioritarias para su alcance y elaborar un plan de implantación de buenas prácticas de manufacturas.

\section{BALANCE DE LA EXPERIENCIA}

En apenas año y medio de operación, esta cooperativa de "segundo piso" ha demostrado ser una eficiente herramienta de inserción en los mercados de la producción de origen campesino.

Como los logros más destacables se pueden reseñar los siguientes:

- En alianza con universidades nacionales, ha desarrollado líneas de productos agroindustriales terminados de alta calidad (cafés tostados y molidos, miel envasada, fríjoles deshidratados, aceites comestibles y cosméticos) que ha insertado en cadenas de supermercados nacionales bajo una marca propia.

- Asimismo, ha conseguido el reconocimiento de entes estatales de promoción del comercio exterior.

- Hoy administra una de las plantas más grandes y modernas de Centroamérica para el procesamiento (descortezado) de semilla de ajonjolí, la planta de Posoltega, tras una negociación de este bien público con el Estado. La empresa cooperativa ha establecido alianzas firmes con empresas agroindustriales nacionales para la comercialización local y en el exterior de productos nicaragüenses de alto valor agregado.

- Algunas cooperativas socias integran una red de turismo sostenible y han iniciado la reconversión de infraestructuras colectivas (casas comunales) en pequeñas instalaciones hoteleras rurales.

El diseño e implantación de sistemas de gestión ha permitido demostrar:

- La exigencia cada vez mayor impuesta por el mercado para facilitar la introducción en él de estos productos. La certificación tanto de los productos como de los sistemas de gestión de las empresas u organi- 
zaciones que los producen, va adquiriendo cada vez una relevancia mayor. Precisamente, ha sido esta exigencia la que ha hecho surgir la necesidad de abordar esta iniciativa.

- Se ha podido constatar la necesidad de formación y capacitación en temas de calidad y certificación existente en los países en desarrollo.

- Estas herramientas son demandadas por organizaciones con un grado de madurez determinado. Podemos destacar la capacidad gestora de la contraparte con la que se ha desarrollado el proyecto como su punto fuerte.

Cuando las organizaciones demandan la implantación de estos sistemas de gestión, estos se convierten en eficaces herramientas de mejora que les ayuda a planificar, ejecutar y gestionar sus procesos fomentando un cambio de mentalidad hacia la mejora continua. El interés de estas herramientas ha quedado demostrado por la continuidad que se le está dando a esta iniciativa.

También se han valorado las mejoras que se obtienen cuando se integran distintos sistemas de gestión y se aprovechan las sinergias existentes entre estos; por ejemplo, los sistemas de gestión de calidad ISO 9001:2008 y el HACCP. Dichas mejoras son:

- Unificar las responsabilidades y los criterios.

- Reducir los costes de implantación, certificación y mantenimiento.

- Reducir el tiempo y los recursos dedicados a las auditorías.

- Reducir la documentación y los registros necesarios, dado que mucha información es común a ambos sistemas; existe un único manual de gestión y los procedimientos generales no se duplican.

- Conseguir mayor eficacia en la formación de sistemas.

- Facilitar la integración de otros sistemas normalizados (por ejemplo, medioambientales).

- Mejorar la imagen de la organización.

- Dotar de visión global y estratégica a la organización.

- Se trata de una oportunidad para iniciar un cambio que mejorará la gestión de la organización. 


\section{HACIA EL FUTURO}

La cooperativa de "segundo piso" objeto de estudio es una organización que ha comenzado a caminar recientemente. Para que en el futuro pueda convertirse en una verdadera alternativa que demuestre lo que puede ser una organización económica y social adecuada a las necesidades de los pequeños productores rurales de Centroamérica, se propone afrontar desafíos como los que se citan a continuación:

a) El control de las cadenas de producción: industrialización y comercialización.- El crecimiento del movimiento cooperativo no se puede limitar a la articulación horizontal de la base productiva, sino al control de los diferentes eslabones en la cadena de agregación de valor. En el nuevo paradigma productivo, es la cadena la que transfiere información y tecnología y, por ende, poder de negociación.

En Nicaragua, frente a las ineficiencias que generan los monopolios de unas pocas familias oligárquicas que controlan ciertos eslabones de las cadenas de producción, el movimiento cooperativo puede oponer un manejo de toda la cadena productiva (desde los insumos hasta el comercio) que disminuya los costos de producción, así como los precios al consumidor, e impacten positivamente en la balanza comercial nacional.

b) La diversificación hacia los servicios.- El movimiento cooperativo agropecuario no puede obviar los otros usos de la tierra que se imponen a los tradicionales. La conciencia sobre esta nueva valorización de la tierra permitirá frenar el desalojo cooperativo. Asimismo, la explotación de los nuevos usos deberá fomentar la generación de empleo, especialmente importante para las generaciones más jóvenes de hijos e hijas de las familias del minifundio agrícola.

En el caso de Nicaragua, dado el amplio control de la tierra en manos de cooperativas y comunidades indígenas, los servicios ambientales (captura de carbono) y aquellos ligados al turismo (siempre que exista un claro marco ético de explotación ambientalmente sostenible, de respeto a la dignidad de los trabajadores y de rescate de la identidad cultural local), pueden ser una fuente agregada de capitalización y fortalecimiento del movimiento.

c) Alianzas con el movimiento cooperativo nacional e internacional: de las redes a las estructuras operativas.- Las redes internacionales creadas sobre la base del intercambio de información y experiencias o de la discusión de problemas comunes (como los tratados de libre 
comercio o el desembarco de los transgénicos) deben ser el origen de la construcción de empresas cooperativas internacionales (e "internacionalistas").

La construcción de estas cooperativas, que superan los ámbitos nacionales, puede dar como resultado soluciones directas e inmediatas a algunos problemas que, como ya se ha indicado en este artículo, limitan el crecimiento cooperativo en los países del Sur.

d) Acceso a los mercados.- Las necesidades logísticas y de información hacen casi imposible la operación eficiente del comercio internacional directo entre las cooperativas del Sur y los canales de distribución de los países enriquecidos. La alianza entre las cooperativas agroindustriales del Sur y las cooperativas de servicios del Norte, en la distribución mayorista primero, y más tarde en la minorista, pueden sustituir la relación tradicional con los grandes importadores por canales más cortos y más eficientes. La adopción de medidas tales como las buenas prácticas de manufacturas, el sistema de análisis de riesgos y puntos críticos de control y el diseño de un sistema de gestión por procesos asegura el cumplimiento de las normas de seguridad, higiene e inocuidad de los alimentos, exigidos en los mercados nacional e internacional.

e) Transferencia tecnológica.- De nuevo, las alianzas internacionales entre movimientos cooperativos locales facilitará la transferencia tecnológica de forma directa y completa, tanto de tecnología "dura" (semillas o equipamiento industrial) como de tecnología "suave" (formas de organización, métodos eficientes de formación cooperativa, etcétera).

\section{REFERENCIAS}

1. Banco Central de Nicaragua (2010). Nicaragua en cifras 2010. Managua: Banco Central de Nicaragua.

2. Banco Mundial (2010). Informe Anual 2010. Washington: Banco Mundial.

3. Blalock, G., Veloso, F. (2007). Imports, productivity and supply chain learning. World Development 35, 1134-1151.

4. Carrasco, J. (2000). Evolución de los enfoques y conceptos de logística. Su impacto en la dirección y gestión de las organizaciones. Economía Industrial, 331, 17-34. 
5. Coque, J., (2005). Compartir soluciones: las cooperativas como factor de desarrollo en zonas desfavorecidas. Madrid: Comité Económico y Social.

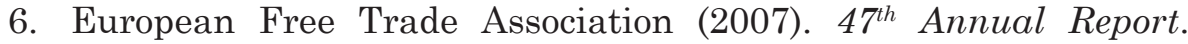
Bruselas: EFTA.

7. Fairtrade Labelling Organizations (2003). A quantum leap in the impact of Fairtrade labelling. FLO's strategic plan. Bonn: FLO.

8. Gereffi, G., Humphrey, J., \& Sturgeon, T., (2005). The governance of global value chains. Review of International Political Economy 1, 78 .

9. International Federation for Alternative Trade (2008). Annual Report 2007. Amsterdam: IFAT.

10. International Organization for Standardization. (2008). Sistemas de gestión de cCalidad ISO 9001:2008. Madrid: Gestión.

11. Londoño, J., \& Mataix, C. (2001). Servicios de apoyo a la microempresa en países en desarrollo. Bilbao: Hegoa.

12. Mataix, C., \& Moyano, C., (2004). La promoción de cooperativas como estrategia para la creación y gestión de cadenas productivas agrarias. Cuadernos Internacionales de Tecnologías para el Desarrollo Humano 4, 45-53.

13. Mutersbaugh, T., (2004). Serve and certify: paradoxes of service work in organic coffee certification. Environment and Planning $22,533-552$.

14. Mutersbaugh, T., (2005). Just-in-space: Certified rural products, labor of quality, and regulatory spaces. Journal of Rural Studies 21, 389-402.

15. Oyarzún, M., (2001). Propuesta de sellos de calidad para productos de la pequeña agroindustria rural en América Latina. Santiago de Chile: FAO.

16. PNUD (2010). Informe sobre desarrollo humano 2010. La verdadera riqueza de las naciones: Caminos al desarrollo humano. Nueva York: PNUD.

17. Raynolds, L. T., (2004). The globalization of organic agro-food networks. World Development 32, 725-743. 
18. Renard, M., (2005). Quality certification, regulation and power in fair trade. Journal of Rural Studies 21, 419-431.

19. Sligo, F. X., \& Massey, C., (2007). Risk, trust and knowledge networks in farmers' learning. Journal of Rural Studies 23, 170182.

20. Verhaegen, I., \& Van Huylenbroeck G., (2001). Costs and benefits for farmers participating in innovative marketing channels for quality food products. Journal of Rural Studies 17, 443-456.

21. Willer, H., \& Yussefi, M., (2005). The world of organic agriculture: statistics and emerging trends. Bonn: IFOAM \& FiBL. 